\title{
Small-amplitude oscillations of magnetization accompanying long-lived spin-precession in ${ }^{3} \mathrm{He}-B$
}

\author{
G. A. Kharadze, N. G. Suramlishvili, and G. E. Vachnadze \\ Institute of Physics of the Georgian Academy of Sciences, 6 Tamarashvili st., Tbilisi GE-380077, Georgia \\ E-mail: khar@physics.iberiapac.ge \\ Submitted January 27, 1997
}

\begin{abstract}
Frequency spectrum of small-amplitude oscillations of magnetization against the background of coherently precessing (phase-correlated) spin modes in superfluid ${ }^{3} \mathrm{He}$ placed in a strong magnetic field depends on the magnitude of spin polarization $S$ and spin-orbit structure of dynamical ordered states. Spectrum of these oscillations is calculated for unconventional precessing modes in ${ }^{3} \mathrm{He}-B$ characterized by $S=S_{0} / 2$, where $S_{0}$ is the value of $S$ at equilibrium.
\end{abstract}

PACS: $67.57 . \mathrm{Lm}$

The coherent dipole-dipole interaction between nuclear magnetic moments in the superfluid phases of liquid ${ }^{3}$ He defines (along with other symmetrybreaking terms) the spin-orbit structure of the equilibrium and dynamic (time-dependent) ordered states of ${ }^{3} \mathrm{He}-A$ and ${ }^{3} \mathrm{He}-B$.

In the presence of a static magnetic field $\mathbf{H}_{0}=H_{0} \hat{z}$ and a low-amplitude transverse rf field of frequency $\omega$ the stable and metastable (longlived) spin-orbit configurations are realized at the minima of the thermodynamic potential

$$
F^{\prime}=F+\omega \mathbf{S}
$$

which is constructed in the coordinate frame rotating with an angular velocity $\boldsymbol{\omega}=\omega \hat{z}$ ( $\mathbf{S}$ denotes the spin-density). In Eq. (1) $F$ is the sum of the Zeeman and the dipole-dipole energies, so that

$$
F=-\omega_{0} \mathbf{S}+U_{D},
$$

where $\boldsymbol{\omega}_{0}=\omega_{0} \hat{z}$ with the Larmor frequency $\omega_{0}=g H_{0}$. Since

$$
F^{\prime}=\left(\omega-\omega_{0}\right) S_{z}+U_{D},
$$

in the absence of the first (spectroscopic) term (at the Larmor resonance $\omega=\omega_{0}$ ) the stationary spinorbit configurations (in the rotating coordinate frame) are defined by the minima of $U_{D}$.

For ${ }^{3} \mathrm{He}-B$

$$
U_{D}=\frac{2}{15} \chi_{B}\left(\Omega_{B} / g\right)^{2}(\operatorname{Sp} \hat{R}-1 / 2)^{2},
$$

where $\chi_{B}$ is the magnetic susceptibility; $\Omega_{B}$ is the frequency of the longitudinal NMR; and the orthogonal matrix $R$ describes the relative rotations of the spin and orbital spaces:

$$
\hat{R}=\hat{R}^{(S)} \hat{R}^{(L)-1} .
$$

Here $\hat{R}^{(S)}$ and $\hat{R}^{(L)}$ are the matrices of $3 D$ rotations of the spin and orbital degrees of freedom, respectively. Parametrizing these rotations by the triples of Euler angles $\left(\alpha_{S}, \beta_{S}, \gamma_{S}\right)$ and $\left(\alpha_{L}, \beta_{L}, \gamma_{L}\right)$, we find

$$
\begin{aligned}
\text { Sp } \hat{R} & \left.=s_{z} l_{z}+\frac{1}{2}\left(1+s_{z}\right)\left(1+l_{z}\right) \cos (\alpha+\gamma)\right)+ \\
& +\frac{1}{2}\left(1-s_{z}\right)\left(1-l_{z}\right) \cos (\alpha-\gamma)+ \\
& +\sqrt{\left(1-s_{z}^{2}\right)\left(1-l_{z}^{2}\right)}(\cos \alpha+\cos \gamma),
\end{aligned}
$$

where $s_{z}=\cos \beta_{S}, l_{z}=\cos \beta_{L}, \alpha=\alpha_{S}-\alpha_{L}$, and $\gamma=\gamma_{S}-\gamma_{L}$

The absolute minimum of (4) is realized at Sp $\hat{R}=1 / 2$. It occurs, in particular, for the nonprecessing states with $s_{z}=1$ and $-1 / 4<l_{z}<1$. The dynamic counterpart (a mirror image $l_{z} \leftrightarrow s_{z}$ ) of this set of equilibrium states is the Brinkman-Smith (BS) spin-precessing mode with $l_{z}=1$ and $-1 / 4<s_{z}<1$. In the BS mode the magnetization is precessing exactly at the Larmor frequency $\omega_{0}$ and the Leggett-Takagi (LT) relaxation mechanism is completely switched off ( $S_{z}=$ const). 
At $\omega \neq \omega_{0}$, where in the rotating coordinate frame the action of the static magnetic field is eliminated only partly, a new hierarchy of the long-lived spin-precessing states is stabilized in ${ }^{3} \mathrm{He}-B$. These states do not correspond to the absolute minimum of $U_{D}$ and the LT relaxation mechanism is operative. Among them the most thoroughly explored is the HPD state $[1,2]$ generated at $\omega>\omega_{0}$. In this situation the spectroscopic term in (3) pushes the left boundary $s_{z}=-1 / 4$ of the BS state farther to the left, and the spin-precessing states with $l_{z}=1$ and $-1<s_{z}<-1 / 4$ are formed at the balance of the dipole-dipole and spectroscopic forces. For the case where $\omega$ exceeds $\omega_{0}$ only slightly the spin-precession takes place at $s_{z} \simeq-1 / 4$ and the LT relaxation is rather slow. Due to the presence of the spectroscopic term, the HPD transforms to the precessing two-domain configuration in the presence of the magnetic field gradient.

An effective way of analyzing other possible long-lived spin-precessing states in ${ }^{3} \mathrm{He}-B$ is to consider the case of a strong magnetic field with $\omega_{0} \gg \Omega_{B}$. In this situation the dynamic variables $\alpha$ and $\gamma$, which appear in expression (4) for the dipole-dipole potential, are naturally arranged as the fast and slow linear combinations (on the long time scale $1 / \Omega_{B}$ ). As a good starting approximation one can discard the rapidly oscillating terms in $U_{D}$ and deal with the average potential $\bar{U}_{D}$ that contains only slow variables: $s_{z}$ and $l_{z}$ and possibly some slow combination of $\alpha$ and $\gamma$. In this way, it is easy to construct a proper first-order solution for the coherently precessing spin-states and then to explore small-amplitude oscillations of magnetization [proportional to $\left(\Omega_{B} / \omega_{0}\right)^{2}<<1$ ], superimposed on the «mean-field» solution.

When constructing the above-described «meanfield» (average) picture, it should be realized that the answer for $\bar{U}_{D}$ depends crucially on the magnitude $S=|\mathbf{S}|$ of the spin-density of a particular precessing state. For the conventional case of $S$ close to its equilibrium value $S_{0}=\left(\chi_{B} / g\right) H_{0}$ a slow angular variable surviving the averaging procedure is $\varphi=\alpha+\gamma$ and corresponding expression for $\bar{U}_{D}$ was extensively used in Ref. 3. The series of homogeneously precessing, metastable states different from HPD were established as a result of establishing a balance between $\bar{U}_{D}$, the spectroscopic term, and the action of the finite-amplitude transverse rf field.

The new metastable spin-precessing states were found in Ref. 4 for unconventional situations with $S=S_{0} / 2$ (the HS state) and $S=2 S_{0}$ (the DS state). In these cases (again for $\omega_{0}>>\Omega_{B}$ )

$$
\begin{gathered}
\bar{U}_{D}=\frac{1}{10} \chi_{B}\left(\Omega_{B} / g\right)^{2}\left[1+2 s_{z}^{2} l_{z}^{2}+\left(l-s_{z}^{2}\right)\left(1-l_{z}^{2}\right)+\right. \\
\left.+\frac{2}{3} \sqrt{\left(1-s_{z}^{2}\right)\left(1-l_{z}^{2}\right)}\left(1+l_{z}\right)\left(1+l_{z}\right) \cos \tilde{\varphi}\right] .
\end{gathered}
$$

where the slow angular variable $\tilde{\varphi}=\alpha+2 \gamma(2 \alpha+\gamma)$ for the HS(DS) state. Considering the case of an exact Larmor resonance $\left(\omega=\omega_{0}\right)$ and noticing that the stationary value $\tilde{\varphi}_{\text {st }}=\pi$, we see that there are two degenerate spin-orbit configurations: $\left(s_{z} \simeq 0.75, l_{z} \simeq 0.3\right)$, and $s_{z} \leftrightarrow l_{z}$. These metastable, coherently precessing, spin states are at the minima of $\bar{U}_{D}$, not at the true minimum of an exact dipole-dipole potential $U_{D}$. For this reason the HS(DS) states, as well as all metastable precessing states considered in Ref. 3, are characterized by the small-amplitude oscillations of the magnetization against the background of the «mean-field» dynamics. These oscillations are driven by the dipole-dipole torque, which is nonzero for the metastable dynamic states. For them the LT relaxation is also operative (as mentioned above, only for the HPD at $\omega \simeq \omega_{0}$ it is anomalously slow because of the closeness to the BS mode). In what follows we study the frequency spectrum of the small-amplitude oscillations of the magnetization, accompanying the longlived (metastable) spin-precessing state in ${ }^{3} \mathrm{He}-B$ with $S=S_{0} / 2$ (HS mode). This question was explored recently by means of the computer simulation [5]. Here we describe the results of an analytic approach based on the self-consistent separation of the fast and slow spin-dynamics appropriate to the strong field case [6] $\left(\omega_{0}>>\Omega_{B}\right)$.

Using two pairs of canonically conjugate variables $(\alpha, P)$ and $(\varphi, S)$ with $\varphi=\alpha+2 \gamma$ and $P=S_{z}-\frac{1}{2} S$, we write the Leggett equations in a dimensionless form

$$
\begin{gathered}
\dot{\alpha}=-1+\varepsilon \frac{\partial f}{\partial P}, \quad \dot{P}=-\varepsilon \frac{\partial f}{\partial \alpha}, \\
\dot{\varphi}=2\left[(S-1 / 2)+\varepsilon \frac{\partial f}{\partial S}\right], \quad \dot{S}=-2 \varepsilon \frac{\partial f}{\partial \varphi},
\end{gathered}
$$

where the time is measured in units of $1 / \omega_{0}$, $(P, S)$ is measured in units of $S_{0}$, and $\varepsilon f=$ $=U_{D} / \omega_{0} S_{0}$ with $\varepsilon=\frac{2}{15}\left(\Omega_{B} / \omega_{0}\right)^{2}<<1$. In order to solve the system of equations (8) to the first order in $\varepsilon$, we use a new set of variables $\bar{\alpha}, \bar{P}, \bar{\varphi}$, and $\bar{S}$ according to the transformation

$$
\begin{aligned}
& \alpha=\bar{\alpha}+\varepsilon u_{\alpha}(\bar{P}, \bar{S}, \bar{\varphi} \mid \bar{\alpha}), \\
& P=\bar{P}+\varepsilon u_{p}(\bar{P}, \bar{S}, \bar{\varphi} \mid \bar{\alpha}),
\end{aligned}
$$




$$
\varphi=\bar{\varphi}+\varepsilon u_{\varphi}, \quad S=\bar{S}+\varepsilon u_{s}
$$

with yet unknown functions $u_{i}$ of slow $(\bar{P}, \bar{S}, \bar{\varphi})$ and fast $(\bar{\alpha})$ variables. In what follows $\bar{\varphi}$ is a slow variable (along with $\bar{P}$ and $\bar{S}$ ) since we consider a «resonating» regime with $S=\frac{1}{2}+\varepsilon \sigma$.

Implying that $\bar{\alpha}, \bar{P}, \bar{S}$, and $\bar{\varphi}$ satisfy a set of equations

$$
\begin{gathered}
\dot{\bar{\alpha}}=-1+\varepsilon A_{\alpha}(\bar{P}, \bar{S}, \bar{\varphi}), \quad \dot{\bar{P}}=\varepsilon A_{p}(\bar{P}, \bar{S}, \bar{\varphi}), \\
\dot{\bar{\varphi}}=\varepsilon\left(2 \sigma+A_{\varphi}(\bar{P}, \bar{S}, \bar{\varphi})\right), \quad \dot{\bar{S}}=\varepsilon A_{s}(\bar{P}, \bar{S}, \bar{\varphi}),
\end{gathered}
$$

we can easily obtain a set of equations for $u_{i}$

$$
\begin{gathered}
\dot{u}_{\alpha}=\frac{\partial f}{\partial \bar{P}}-A_{\alpha}, \quad \dot{u}_{p}=-\frac{\partial f}{\partial \bar{\alpha}}-A_{p}, \\
\dot{u}_{\varphi}=2\left(\frac{\partial f}{\partial \bar{S}}+u_{s}\right)-A_{\varphi}, \quad \dot{u}_{s}=-2 \frac{\partial f}{\partial \bar{\varphi}}-A_{s} .
\end{gathered}
$$

Here the functions $A_{i}$ of the slow variables $\bar{P}, \bar{S}$, and $\bar{\varphi}$ are found according to the condition for the absence of secular terms in the solution of Eqs. (11) $\left(\lim _{\bar{\alpha} \rightarrow \infty}\left|u_{i}\right|<\infty\right)$. In this way it can be established that

$$
\begin{gathered}
A_{\alpha}=\frac{\partial \bar{f}}{\partial \bar{P}}, \quad A_{p}=-\frac{\partial \bar{f}}{\partial \bar{\alpha}}, \\
A_{\varphi}=2\left(\frac{\partial \bar{f}}{\partial \bar{S}}+\bar{u}_{s}\right), \quad A_{s}=-2 \frac{\partial \bar{f}}{\partial \bar{\varphi}},
\end{gathered}
$$

where $\bar{f}$ and $\bar{u}_{s}$ denote time-averaged parts of the corresponding functions of the fast variable $\bar{\alpha}$ (without loss of generality it can be assumed that all $\bar{u}_{i}=0$ ). Isolating a rapidly oscillating part of the dipole-dipole energy $\tilde{f}=f-\bar{f}$ and noticing that $\dot{u}_{i} \simeq\left(\partial u_{i} / \partial \bar{\alpha}\right) \dot{\bar{\alpha}} \simeq-\partial u_{i} / \partial \bar{\alpha}$, from Eqs. (11) we find that

$$
\begin{gathered}
u_{\alpha}(\bar{P}, \bar{S}, \bar{\varphi} \mid \bar{\alpha})=-\int \frac{\partial \tilde{f}}{\partial \bar{P}} d \bar{\alpha} \\
u_{p}(\bar{P}, \bar{S}, \bar{\varphi} \mid \bar{\alpha})=\int \frac{\partial \tilde{f}}{\partial \bar{\alpha}} d \bar{\alpha}
\end{gathered}
$$

$$
u_{s}(\bar{P}, \bar{S}, \bar{\varphi} \mid \bar{\alpha})=2 \int \frac{\partial \tilde{f}}{\partial \bar{\varphi}} d \bar{\alpha}
$$

Stationary values of the variables $\bar{P}, \bar{S}$, and $\bar{\varphi}$ are found according to the equations

$$
\frac{\partial \bar{f}}{\partial \bar{\alpha}}=0, \quad \frac{\partial \bar{f}}{\partial \bar{\varphi}}=0, \quad \frac{\partial \bar{f}}{\partial \bar{S}}=-\sigma .
$$

The first of these equations is satisfied identically since $\bar{f}$ is independent of $\bar{\alpha}$. The second one shows that the stationary value $\bar{\varphi}_{\text {st }}=\pi$ and from the last one we see that

$$
\bar{S}_{\mathrm{st}}=\frac{1}{2}-\varepsilon \frac{\partial \bar{f}}{\partial \bar{S}} .
$$

From the relation $S_{z}=P+1 / 2 S$ we conclude that the low-amplitude high-frequency oscillations of $S_{z}$ and $S_{\perp}$ are given by

$$
\begin{gathered}
\delta S_{z}=S_{z}-\bar{S}_{z}=\varepsilon u_{z}(\bar{\alpha}), \\
\delta S_{\perp}=S_{\perp}-\bar{S}_{\perp}=\varepsilon u_{\perp}(\bar{\alpha}),
\end{gathered}
$$

where

$$
\begin{gathered}
u_{z}(\bar{\alpha})=u_{p}(\bar{\alpha})+\frac{1}{2} u_{s}(\bar{\alpha}) \\
u_{\perp}(\bar{\alpha})=\frac{1}{\sqrt{1-s_{z}^{2}}}\left(u_{s}(\bar{\alpha})-u_{z}(\bar{\alpha}) s_{z}\right) .
\end{gathered}
$$

Now we can easily pass to $S_{x}$ and $S_{y}$ components:

$$
\begin{aligned}
& S_{x}=\bar{S}_{\perp} \cos \bar{\alpha}+\varepsilon\left(u_{\perp}(\bar{\alpha}) \cos \bar{\alpha}-\bar{S}_{\perp} u_{\alpha}(\bar{\alpha}) \sin \bar{\alpha}\right) \\
& S_{y}=S_{\perp} \sin \bar{\alpha}+\varepsilon\left(u_{\perp}(\bar{\alpha}) \sin \bar{\alpha}+\bar{S}_{\perp} u_{\alpha}(\bar{\alpha}) \cos \bar{\alpha}\right) .
\end{aligned}
$$

In order to obtain the final results for $\delta S_{z}, \delta S_{x}$, and $\delta S_{y}$ we must construct, according to Eqs. (13), the oscillatory contributions $u_{\alpha}(\bar{\alpha}), u_{p}(\bar{\alpha})$, and $u_{s}(\bar{\alpha})$. After some algebra, from Eqs. (4), (6), and (7) it can be shown that

$$
\begin{aligned}
& \tilde{f}(\alpha)=\frac{1}{8}\left[\left(1+s_{z}\right)^{2}\left(1+l_{z}\right)^{2} \cos (\alpha+\varphi)+\left(1-s_{z}\right)^{2}\left(1-l_{z}\right)^{2} \cos (3 \alpha-\varphi)\right]+ \\
& +\frac{1}{2} \sqrt{\left(1-s_{z}^{2}\right)\left(1-l_{z}^{2}\right)}\left[\left(1+s_{z}\right)\left(1+l_{z}\right)\left(\cos \alpha+\cos \frac{\alpha-\varphi}{2}+\cos \frac{3 \alpha+\varphi}{2}\right)+\right. \\
& \left.\quad+\left(1-s_{z}\right)\left(1-l_{z}\right)\left(\cos \alpha+\cos \frac{\alpha-\varphi}{2}+\cos (2 \alpha-\varphi)+\cos \frac{5 \alpha-\varphi}{2}\right)\right]+
\end{aligned}
$$




$$
\begin{aligned}
+\frac{3}{4}\left(1-s_{z}^{2}\right)\left(1-l_{z}^{2}\right)\left[\cos 2 \alpha+\cos (\alpha-\varphi)+\frac{4}{3} \cos \frac{\alpha+\varphi}{2}+\frac{4}{3} \cos \frac{3 \alpha-\varphi}{2}\right]+ \\
+\left(s_{z} l_{z}-1 / 2\right)\left[\left(1+s_{z}\right)\left(1+l_{z}\right) \cos \frac{\alpha+\varphi}{2}+\left(1-s_{z}\right)\left(1-l_{z}\right) \cos \frac{3 \alpha-\varphi}{2}+\right. \\
\left.+2 \sqrt{\left(1-s_{z}^{2}\right)\left(1-l_{z}^{2}\right)}\left(\cos \alpha+\cos \frac{\alpha-\varphi}{2}\right)\right]
\end{aligned}
$$

Using this expression, after some lengthy calculations, we find

$$
\begin{gathered}
u_{z}(\bar{\alpha})= \\
=\sum_{n=1}^{3}\left[a_{n}\left(s_{z}, l_{z}\right) \cos n \bar{\alpha}+a_{n-1 / 2}\left(s_{z}, l_{z}\right) \sin (n-1 / 2) \bar{\alpha}\right] \\
u_{\perp}(\bar{\alpha})= \\
=\sum_{n=1}^{3}\left[b_{n}\left(s_{z}, l_{z}\right) \cos n \bar{\alpha}+b_{n-1 / 2}\left(s_{z}, l_{z}\right) \sin (n-1 / 2) \bar{\alpha}\right]
\end{gathered}
$$

and

$$
\begin{gathered}
u_{\alpha}(\bar{\alpha})=\frac{1}{\sqrt{1-s_{z}^{2}}} \times \\
\times \sum_{n=1}^{3}\left[c_{n}\left(s_{z}, l_{z}\right) \sin n \bar{\alpha}+c_{n-1 / 2}\left(s_{z}, l_{z}\right) \cos (n-1 / 2) \bar{\alpha}\right],
\end{gathered}
$$

where the coefficients $a, b$, and $c$ are given in the Appendix.

According to Eqs. (16) and (18) we finally arrive to the following answer for the small-amplitude oscillating components $\delta S_{z}, \delta S_{x}$, and $\delta S_{y}$ :

$$
\begin{gathered}
\delta S_{z}(t)=\varepsilon \sum_{n=1}^{3}\left[a_{n} \cos n \bar{\alpha}+a_{n-1 / 2} \sin (n-1 / 2) \bar{\alpha}\right], \\
\delta S_{x}(t)=\frac{1}{2} \varepsilon \sum_{n-1}^{3}\left[d_{n}^{(+)} \cos (n+1) \bar{\alpha}+d_{n}^{(-)} \cos (n-1) \bar{\alpha}+\right. \\
\left.+d_{n-1 / 2}^{(-)} \sin (n+1 / 2) \bar{\alpha}+d_{n-1 / 2}^{(+)} \sin (n-3 / 2) \bar{\alpha}\right], \\
\delta S_{y}(t)=\frac{1}{2} \varepsilon \sum_{n=1}^{3}\left[d_{n}^{(+)} \sin (n+1) \bar{\alpha}-d_{n}^{(-)} \sin (n-1) \bar{\alpha}+\right.
\end{gathered}
$$

$$
\left.+d_{n-1 / 2}^{(+)} \cos (n-3 / 2) \bar{\alpha}-d_{n-1 / 2}^{(-)} \cos (n+1 / 2) \bar{\alpha}\right]
$$

where $d^{( \pm)}=b \pm 1 / 2 c$.

Inspection of Eq. (23) shows that the spectrum of longitudinal oscillations of magnetization superimposed on the HS precessing mode comprises highfrequency harmonics with $\tilde{\omega} \simeq \omega_{0}, 2 \omega_{0}, 3 \omega_{0}$, $1 / 2 \omega_{0}, 3 / 2 \omega_{0}$, and $5 / 2 \omega_{0}$. The same set of harmonics governs the small-amplitude oscillations of transversal components of the precessing magnetization [see Eqs. (24) and (25), keeping in mind that $\left.d_{3}^{(+)}=d_{5 / 2}^{(-)}=0\right]$.

It should be stressed that the coefficients $a\left(s_{z}, l_{z}\right)$ are symmetric with respect to the interchange $s_{z} \leftrightarrow l_{z}$ (see the Appendix), so that the «mirror» precessing HS states found in Ref. 4 for the case of a Larmor resonance $\left(\omega=\omega_{0}\right)$ are characterized by identical longitudinal oscillations. In contrast to this observation, the coefficients entering the expressions for $\delta S_{x}$ and $\delta S_{y}$ have no spin-orbit symmetry and measurement of the spectrum of transverse small-amplitude oscillations in the case of HS mode at $\omega=\omega_{0}$ can discriminate between the two above-mentioned degenerate spin-orbit states. It can be checked that in the case of the precessing mode I $\left(s_{z} \simeq 0.75, l_{z} \simeq 0.3\right)$ the oscillations with $\tilde{\omega}=3 / 2 \omega_{0}$ have the largest amplitude. Next are contributions of $\tilde{\omega}=\left(1 / 2 \omega_{0}, \omega_{0}\right)$, which have about four times smaller amplitudes. In the case of the precessing mode II $\left(s_{z} \simeq 0.3, l_{z} \simeq 0.75\right)$ the oscillations with $\tilde{\omega}=3 / 2 \omega_{0}$ are again the strongest, but now contributions of comparable amplitudes come from $\tilde{\omega}=\left(1 / 2 \omega_{0}, 2 \omega_{0}\right)$.

Away from the Larmor resonance $\left(\omega \neq \omega_{0}\right)$ the states I and II are displaced from their initial positions. In particular, as has been demonstrated in Ref. 7 , in the presence of sufficiently large negative spectroscopic term $\left(\left(\omega<\omega_{0}\right)\right)$ the state I moves toward a spin-orbit configuration $s_{z} \simeq 1, l_{z} \simeq 0$, while the state II loses its stability. Expressions for $\delta S_{z}, \delta S_{x}$, and $\delta S_{y}$ are especially simple for this HS precessing state: 


$$
\begin{gathered}
\delta S_{z}(t)=-\varepsilon(\cos \bar{\alpha}-2 \sin \bar{\alpha} / 2), \\
\delta S_{x}(t)=\frac{2}{3} \varepsilon \sin \bar{\alpha} / 2 \\
\delta S_{y}(t)=\frac{2}{3} \varepsilon \cos \bar{\alpha} / 2 .
\end{gathered}
$$

The HS coherently precessing mode in the spinorbit configuration $s_{z} \simeq 1, l_{z} \simeq 0$ was recently observed experimentally [8]. It has been identified by means of application of a transverse $90^{\circ} \mathrm{rf}$ pulse, after which the measurement of the amplitude of an induction signal has allowed to establish the magnitude of the spin polarization of the precessing state. It is certainly the most direct way to identify a spin-precessing mode with $S=S_{0} / 2$ and $s_{z} \simeq 1$. At the same time, the spectroscopy of small-amplitude oscillations of magnetization accompaning phase-correlated spin-precessing states could serve as an additional source of information about their spin-orbit structure.

We would like to thank Prof. V. Dmitriev for providing us with his recent experimental results prior to publication.

\section{Appendix}

We give here the expressions for the coefficients appearing in Eqs. (23)-(25). We introduce the transverse components $s_{\perp}=\sqrt{1-s_{z}^{2}}$ and $l_{\perp}=\sqrt{1-l_{z}^{2}}$ :

$$
\begin{gathered}
a_{1}=3 s_{z} s_{\perp} l_{z} l_{\perp}-\frac{1}{4}\left(1+s_{z}\right)^{2}\left(1+l_{z}\right)^{2} \\
a_{2}=\frac{1}{4}\left[3\left(s_{\perp} l_{\perp}\right)^{2}-s_{\perp}\left(1-s_{z}\right) l_{\perp}\left(1-l_{z}\right)\right] \\
a_{3}=-\frac{1}{12}\left(s_{\perp} l_{\perp}\right)^{2} \\
a_{1 / 2}=-4\left(1+s_{z}\right)\left(1 / 2-s_{z}\right)\left(1+l_{z}\right)\left(1 / 2-l_{z}\right) \\
a_{3 / 2}=-\frac{2}{3}\left[s_{\perp}\left(1+s_{z}\right) l_{\perp}\left(l+l_{z}\right)-\right. \\
\left.-2\left(1-s_{z}\right)\left(1 / 2+s_{z}\right)\left(1-l_{z}\right)\left(1 / 2+l_{z}\right)\right] \\
a_{5 / 2}=\frac{2}{5} s_{\perp}\left(1-l_{z}\right) l_{\perp}\left(1-l_{z}\right) .
\end{gathered}
$$

These coefficients have spin-orbit symmetry (they are invariant at $s_{z} \leftrightarrow l_{z}$ ). On the other hand, the coefficients $b$ and $c$ have no such symmetry:

$$
\begin{gathered}
b_{1}=\frac{1}{2} s_{\perp}\left[3 l_{\perp}^{2}-\frac{1}{2}\left(1+s_{z}\right)\left(1+l_{z}\right)^{2}\right]-3 s_{z}^{2} l_{\perp} l_{z}, \\
b_{2}=\frac{1}{4}\left[\left(2+s_{z}\right)\left(1-s_{z}\right) l_{\perp}\left(1-l_{z}\right)-3 s_{\perp} s_{z} l_{\perp}^{2}\right], \\
b_{3}=\frac{1}{12} s_{\perp}\left(1-s_{z}\right)\left(1-l_{z}\right)^{2}, \\
b_{1 / 2}=-2\left[3 s_{z} l_{\perp} l_{z}+2 s_{\perp}\left(1 / 2-s_{z}\right)\left(1+l_{z}\right)\left(1 / 2-l_{z}\right)\right], \\
b_{3 / 2}=-\frac{2}{3}\left[\left(1+s_{z}\right)\left(1 / 2-s_{z}\right) l_{\perp}\left(1+l_{z}\right)+\right. \\
\left.+2 s_{\perp}\left(1 / 2+s_{z}\right)\left(1-l_{z}\right)\left(1 / 2+l_{z}\right)\right], \\
b_{5 / 2}=-\frac{2}{5}\left(1-s_{z}\right)\left(1 / 2+s_{z}\right)\left(1-l_{z}\right) l_{\perp} ; \\
c_{\perp}\left[\frac{1}{2}\left(1+s_{z}\right)\left(1+l_{z}\right){ }^{2}-3 s_{z} l_{\perp}^{2}\right]-6\left(1-2 s_{z}^{2}\right) l_{\perp} l_{z}, \\
c_{2}=\frac{3}{2} s_{\perp} s_{z} l_{\perp}^{2}-\left(1-s_{z}\right)\left(1 / 2+s_{z}\right) l_{\perp}\left(1-l_{z}\right), \\
c_{3 / 2}=\frac{4}{3}\left[s_{\perp}\left(1-4 s_{z}\right)\left(1-l_{z}\right)\left(1 / 2+l_{z}\right)-\right. \\
-\left(1+s_{z}\right)\left(1 / 2-s_{z}\right)\left(l_{\perp}\left(1+l_{z}\right)\right], \\
c_{1 / 2}=4\left[3\left(1-2 s_{z}^{2}\right) l_{\perp} l_{z}+\right. \\
\left.+s_{\perp}\left(1+4 s_{z}\right)\left(1+l_{z}\right)\left(1 / 2-l_{z}\right)\right],
\end{gathered}
$$

1. Yu. M. Bunkov, in: Progress in Low Temperature Physics, v. 24, W. P. Halperin (ed.), Elsevier Science (1995), p. 69 .

2. I. A. Fomin, in: Modern Problems of Condensed Matter Sciences, v. 26: Helium Three W. P. Halperin and L. P. Pitaevskii (eds.), North Holland, Amsterdam (1990), p. 610 .

3. G. E. Volovik, J. Phys.: Condens. Matt. 5, 1759 (1993).

4. G. Kharadze and G. Vachnadze, JETP Lett. 56, 458 (1992).

5. V. V. Dmitriev, private communication.

6. I. A. Fomin, JETP 50, 144 (1979).

7. G. E. Vachnadze and N. G. Suramlishvili, Low Temp. Phys. 22, 545 (1996).

8. V. V. Dmitriev et al., Phys. Rev. Lett. 7, 86 (1997). 